\title{
From syntrophic bacteria to beyond SAGD! The origin and impact of oil viscosity variations in heavy oil reservoirs and routes to low carbon emission energy recovery
}

\author{
Steve Larter, ${ }^{1,2}$ Barry Bennett $^{2}$ and Ian Gates ${ }^{1,2}$ \\ ${ }^{I} P R G$, University of Calgary, Canada. \\ ${ }^{2}$ Gushor Inc., Calgary, Canada.
}

The recently discovered anaerobic biological conversion of liquid petroleum hydrocarbons to methane in oil reservoirs (methanogenesis), on geological timescales, occurs through biological action by syntrophic bacteria and methanogenic archaea at temperatures as low as $15^{\circ} \mathrm{C}(\mathrm{Head}$ et al., 2003; Jones et al., 2008). As the oil is destroyed from the bottom of the oil column, large vertical and lateral gradients in oil viscosity are formed which impact optimal placements of wells for oil recovery (Larter et al, 2008). Understanding the biodegradation process that produces heavy oil and methane offers great potential both to improve recovery of existing heavy oil resources, through more efficient use of energy during oil recovery and also, potentially, to move away from oil recovery as the major energy process entirely! The discovery of the MADCOR process (Methanogenic Alkane Degradation by Carbon Dioxide Reduction), as it has been termed, raises many possibilities for reduced emission to atmosphere energy recovery (REAR) processes as methane is quantitatively produced from biodegraded alkanes, utilising water as co-reactant, with large amounts of molecular hydrogen being a principal intermediate (Jones et al., 2008).

Microbially produced reservoir fluid heterogeneities are ubiquitous in heavy oil and tar sand (HOTS) reservoirs and impact reservoir processes such as steam floods, SAGD or CSS that depend on uniform oil mobility for optimal recovery(Larter et al, 2008). They also impact cold recovery protocols. These variations, together with reservoir heterogeneities, can impact optimal recovery process design, well placements and field management. Here we discuss the use of integrated fluid and reservoir characterisation coupled with optimised reservoir engineering solutions and our improved understanding of heavy oil origin to reduce emissions and energy requirements. In addition, in heavy oil reservoirs, varying oil composition documents mass transport efficiency within a reservoir and can be used to assess reservoir connectivity and during production, to chemically monitor the progress of production across a reservoir. For any reservoir, customisation of well placements and operating strategies to measured reservoir and fluid hetero-geneities, which we call geotailoring, greatly improves predicted recovery performance. Similarly, recovery processes designed for more sustainable recovery of HOTS including optimised well configurations and production strategies that work with observed geological barriers and oil mobility gradients of natural reservoirs, result in greater geotolerance and energy efficiency with reduced emissions. We describe such processes.

1. Head, I.M., Jones, D.M., Larter, S.R. (2003). Biological activity in the deep subsurface and the origin of heavy oil. Nature $\mathbf{4 2 6} 344-352$.

2. Jones, D. M., Head, I. M., Gray, N. D., Adams, J. J., Rowan, A. K., Aitken, C. M., Bennett, B., Huang, H., Brown, A., Bowler, B. F. J., Oldenburg, T., Erdmann, M., Larter, S. R. (2008). Crude-oil biodegradation via methanogenesis in subsurface petroleum reservoirs. Nature 451, 176-180.

3. Larter, S., Adams, J., Gates, I. D., Bennett, B., Huang, H. (2008). The origin, prediction and impact of oil viscosity heterogeneity on the production characteristics of tar sand and heavy oil reservoirs. Journal of Canadian Petroleum Technology, 47, 52-61. 